\title{
Long-Term Cholic Acid Treatment in a Patient with Zellweger Spectrum Disorder
}

\author{
James E. Heubi ${ }^{a} \quad$ Warren P. Bishop ${ }^{b}$ \\ aDivisions of Pediatric Gastroenterology, Hepatology and Nutrition and Pathology and \\ Laboratory Medicine, Cincinnati Children's Hospital Medical Center, University of \\ Cincinnati, Cincinnati, OH, USA; bPediatric Gastroenterology, Carver College of Medicine, \\ University of lowa, lowa City, IA, USA
}

\section{Keywords}

Peroxisomal biogenesis disorder - Bile acid synthesis - Hepatocellular carcinoma - Neonatal adrenoleukodystrophy

\begin{abstract}
Zellweger spectrum disorders (ZSDs) are a subgroup of peroxisomal biogenesis disorder s with a generalized defect in peroxisome function. Liver disease in ZSDs has been associated with the lack of peroxisomal $\beta$-oxidation of $\mathrm{C}_{27}$-bile acid intermediates to form primary $\mathrm{C}_{24}$-bile acids, which prevents normal physiologic feedback and leads to accumulation of hepatotoxic bile acid intermediates. Primary bile acid therapy, oral cholic acid (CA), as adjunctive treatment for ZSDs, restores physiologic feedback inhibition on bile acid synthesis and inhibits formation of hepatotoxic bile acid intermediates. Our patient is a Caucasian male diagnosed with moderately severe ZSD at age 5 months, and he received long-term CA therapy from age 16 months through 19 years old. CA treatment was well tolerated, with no reports of adverse events. His liver biopsy prior to CA therapy showed cholestasis, periportal inflammation, and bridging fibrosis. Following 5 months of CA therapy, his liver biopsy showed improvement in
\end{abstract}


inflammation and no change in fibrosis. Serum liver enzymes during CA therapy improved compared to pre-therapy levels but frequently were above the upper limit of normal. At age 19 years, following several years with clinical cirrhosis with severe portal hypertension, he presented with worsening jaundice, and he was diagnosed with hepatocellular cancer (HCC). Earlyonset advanced liver disease associated with ZSD and natural disease progression that is not completely suppressed with CA treatment likely caused HCC in our patient. Greater awareness is needed of the possibility of development of HCC in patients with moderately severe ZSD who survive past childhood.

\section{Introduction}

Zellweger spectrum disorders (ZSDs; severe OMIM \#214100, mild OMIM \#601539) are a subgroup within peroxisomal biogenesis disorders. ZSDs have a generalized defect in peroxisome function that impairs fatty acid $\beta$-oxidation and bile acid synthesis, reduces bile flow due to reduced $\mathrm{C}_{24}$-primary bile acids, and results in accumulation of hepatotoxic $\mathrm{C}_{27}$-bile acids (dihydroxycholestanoic and trihydroxycholestanoic acid), potentially leading to progressive liver injury $[1,2]$. Patients across the ZSD severity spectrum show a wide range of clinical symptoms, including mild to moderate or severe developmental delay, neurological abnormalities, vision and hearing impairment, liver dysfunction, and hepatomegaly [3]. Patient survival varies by ZSD severity, with mild ZSD patients surviving into adulthood, patients with moderate ZSD possibly surviving to their teens, and patients with the most severe form of ZSD typically not surviving beyond their first year [1].

The dysregulation of bile acid synthesis in ZSDs derives from the absence of peroxisomal $\beta$-oxidation of $\mathrm{C}_{27}$-bile acid intermediates, which prevents side-chain oxidation and formation of normal $\mathrm{C}_{24}$-primary bile acids, leading to the loss of normal feedback regulation of bile acid synthesis [2]. The resulting further production and accumulation of $\mathrm{C}_{27}$-bile acid intermediates has been linked with progressive liver disease [4]. Adjunctive treatment of ZSD with primary bile acid, oral cholic acid (CA; Cholbam ${ }^{\circledR}$, Retrophin, Inc., San Diego, CA, USA), restores physiologic feedback inhibition on bile acid synthesis by activation of the nuclear receptor farnesoid X receptor (FXR) [5]. This reduces transcriptional activation of cholesterol $7 \alpha$-hydroxylase (CYP7A1), the rate-limiting enzyme in bile acid biosynthesis. The result is the inhibition of formation of hepatotoxic $\mathrm{C}_{27}$-bile acid intermediates from cholesterol [4, 5]. Downregulation of bile acid synthesis with reduction in levels of $\mathrm{C}_{27}$-bile acid intermediates during CA therapy was first demonstrated more than 20 years ago in an infant with severe ZSD [6] and has been shown more recently in ZSD patients following CA treatment for 9 months [2] and during long-term CA therapy [7].

\section{Case Report}

We present the long-term CA treatment of a male Caucasian diagnosed at age 5 months with moderately severe ZSD (formerly known as neonatal adrenoleukodystrophy), with development of hepatocellular carcinoma (HCC) at age 19 years. Our patient was initially treated 


\section{Case Reports in Gastroenterology}

Case Rep Gastroenterol 2018;12:661-670

(c) 2018 The Author(s). Published by S. Karger AG, Basel www.karger.com/crg

Heubi and Bishop: Long-Term Cholic Acid Treatment in a Patient with Zellweger Spectrum Disorder

with CA within an investigator-initiated compassionate use program (NCT00007020) that was followed by an open-label, nonrandomized, noncomparative clinical study (NCT01438411). These studies were approved by the Institutional Review Board of the Cincinnati Children's Hospital Medical Center, and the patient's parents provided informed consent for inclusion. Within these studies, our patientreceived clinical care from his local health care providers and data collection was not required at standardized time points. Study-related laboratory assessments included the serum liver chemistriesalanine aminotransferase (ALT), alkaline phosphatase (ALP), aspartate aminotransferase (AST), and bilirubin. Additionally, a percutaneous liver biopsy was completed before initiation of CA treatment and 5 months following onset of CA treatment. Mass spectralanalysis of urinary bile acids was performed, with fast atom bombardment ionization-mass spectrometry (FAB-MS) at the time of study entry to verify eligibility for study inclusion. FAB-MS was repeated throughout the study to monitor atypical urinary bile acid levels and used semi-quantitative assessment of normal, slight/mild, or significant/moderate increased levels of atypical bile acids.

At ZSD diagnosis, our patient had cholestasis with elevated urinary bile acids and markedly elevated serum liver enzymes but with a normal serum bilirubin (Table 1). Two liver biopsies were performed before CA therapy. The first biopsy (age 4 months) had findings consistent with cholestasis, hepatocellular necrosis with presence of giant cells, periportal inflammation, and moderate fibrosis. The second biopsy (age 16 months) had reduced cholestasis, but with persistent hepatocellular necrosis, periportal inflammation, and progression to bridging fibrosis.

He received CA therapy $(135 \mathrm{mg} /$ day $[10 \mathrm{mg} / \mathrm{kg} /$ day], increased to $300 \mathrm{mg} /$ day [15 mg/ $\mathrm{kg} /$ day]) over the course of 18 years and 6 months, beginning at age 16 months. No treatmentrelated adverse effects were reported. Liver biopsy following 5 months of CA therapy showed improvement in inflammation and no change in fibrosis (Fig. 1a). He showed normalization of urinary bile acids from CA initiation through age 4 years, followed by moderate elevation of urinary bile acids between ages 5 and 6 years, and a return to slight/mild elevation or normalized urinary bile acids thereafter (Fig. 2). He demonstrated profound developmental disability with no change during CA therapy.

Serum liver enzymes during CA therapy were lessabnormal compared with pre-CA levels but frequently were above the upper limit of normal (Table 1). The last available serum liver chemistries, following 18.5 years of CA therapy, were obtained when he presented with worsening jaundice, was diagnosed with HCC (Fig. 1b), and died 1 month later. For years prior to HCC diagnosis, he had clinical cirrhosis with severe portal hypertension, manifested by splenomegaly and esophageal varices requiring treatment with variceal band ligation and propranolol.

\section{Discussion/Con clusion}

In ZSD patients, development of chronic liver disease involving fibrosis and cirrhosis requires patient survival that is long enough for the advancement of the liver disease [8]. HCC in our 19-year-old patient was likely caused by his early-onset advanced liver disease (such as cirrhosis, a key risk factor for HCC [9]) and by potential carcinogenic effects of the natural disease progression of ZSD (such as accumulating dihydroxycholestanoic and trihy droxycho- 


\section{Case Reports in Gastroenterology}

Case Rep Gastroenterol 2018;12:661-670

DOI: 10.1159/000494555

(c) 2018 The Author(s). Published by S. Karger AG, Basel www.karger.com/crg

Heubi and Bishop: Long-Term Cholic Acid Treatment in a Patient with Zellweger Spectrum Disorder

lestanoic acid [5]; Fig. 3) that are not completely suppressed with CA treatment [10], as shown in the intermittent urinary bile acid elevations in our patient. To our knowledge, there are no prior published reports of HCC in ZSD patients. This is surprising as the natural disease progression may increase HCC risk, as indicated by mouse models of peroxisome biogenesis defects [11]. As depicted in the overview of liver metabolic abnormalities in mice with peroxisome biogenesis defects shown in Figure 3, metabolite alterations may be a direct consequence of peroxisome inactivity (e.g., bile acids) or secondary due to activation of proliferatoractivated receptor alpha target genes, altered cholesterol homeostasis, and energy metabolism. Together this results in steatosis and steatohepatitis. The hypothesized increa sed carcinogenesis risk occurs through multiple metabolic deregulation pathways involving hepatotoxicity of accumulating $\mathrm{C}_{27}$-bile acid intermediates (e.g., dihydroxycholestanoic and trihydroxycholestanoic acid) and pathways independent of bile acids, such as proliferator-activated receptor alpha activation and increased endoplasmic reticulum stress [11]. Additionally, in another example of increased HCC risk as the ZSD disease progresses, a ZSD mouse model with hepatocyte-selective elimination of peroxisomes showed early severe hepatomegaly and fibrosis and extensive liver tumors by 12 months [12].

$\mathrm{C}_{27}$-bile acid intermediates may also play an important role in the neurological symptoms associated with ZSDs. It is hypothesized that $\mathrm{C}_{27}$-bile acid intermediates cross the blood-brain barrier and damage the central nervous system [3]. In support of this hypothesis, brain tissues from patients with a peroxisome deficiency disorder were found to have increased $\mathrm{C}_{27}$-bile acids, specifically unconjugated dihydroxycholestanoic and trihydroxycholestanoic acid, compared with controls [5]. Further, ZSD mouse models have shown impairments in developing neocortex and cerebellum and in brain neuronal migration [13], and bile acid treatment partially restored cerebellar abnormalities [14].

Our experience with CA therapy in this patient with advanced liver disease differed from the 4 ZSD patients with advanced liver disease reported by Berendse et al. [2]. In contrast to the poor response seen by Berendse, our patient responded to CA treatment with a reduction in serum aminotransferasesand initial normalization of urinary bile acids. No hepatotoxic effects were evident, and serum bilirubin remained normal until the onset of HCC. A possible explanation for the improved response in our patient is his younger age at CA treatment in itiation (16 months old vs. the 4 - to 10-year-old patients described by Berendse et al. [2]).

Long-term CA therapy appears to have been well tolerated in our patient with moderately severe ZSD without report of side effects and with continuation of therapy over 18 years. In our patient case, treatment-related adverse events were not systematically collected. Rather, side effects were reported by the patient's family as needed. The registration trial that supported FDA approval of CA was conducted over 18 years and included patients with ZSD and bile acid synthesis disorders due to single-enzyme defects. Within the registration trial, the most common treatment-emergent adverse events were diarrhea, urinary tract infection, and dehydration [7].

Early, long-term CA therapy in ZSD patients may help sustain liver function, as suggested by a significant increase in the percentage of ZSD patients showing normalized urinary bile acids and serum liver enzyme levels that stabilized or were within normal limits following long-term treatment in the registration trial that supported FDA approval of CA [7], including 3 patients whose individual CA treatment history and liver-related outcomes over long-term CA therapy were recently reported [15]. Liver synthetic function in our patient reported here 
Heubi and Bishop: Long-Term Cholic Acid Treatment in a Patient with Zellweger Spectrum Disorder

was maintained during 18 years of CA therapy, but CA treatment did not affect his profound neurologic injury or prevent development of portal hypertension, clinical cirrhosis, and HCC. Our patient survived to an older age than is typically reported for patients with moderately severe ZSD. Whether CA therapy contributed to his survival could not be determined in this patient. Further studies are needed, particularly in patients with early initiation of treatment, to elucidate the potential long-term hepatic and extrahepatic effects of CA therapy. Early-onset advanced liver disease associated with his moderately severe ZSD and natural disease progression that is not completely suppressed with CA treatmentlikely caused HCC in our patient. Greater awareness is needed of the possibility of development of HCC in patients with moderately severe ZSD who survive past childhood.

\section{Acknowledgement}

The authors thank Kevin E. Bove, MD, for providing the interpretation of the liver biopsy histology image and the liver MRI for this paper.

\section{Statement of Ethics}

The CA treatment that provided the data for this case report was under an FDA IND and was approved by the IRB of the Cincinnati Children's Hospital Medical Center. All procedures were in accordance with the ethical standards of the responsible committee on human experimentation (institutional and national) and with the Helsinki Declaration of 1975, as revised in 2000. The patient's parents provided informed consent for inclusion in the FDA IND studies and in this report.

\section{Disclosure Statement}

J.E.H.: consultant, Alnylam, FrieslandCampina, Nordmark, Retrophin, Shire; equity interest, Asklepion. W.P.B.: No disclosures to report.

\section{Funding Sources}

Writing and editorial support was provided by Lynanne McGuire, PhD, of MedVal Scientific Information Services, LLC, of Princeton, NJ, and was funded by Retrophin, Inc. The manuscript was prepared according to the International Society for Medical Publication Professionals' "Good Publication Practice for Communicating Company-Sponsored Medical Research: The GPP3 Guidelines." 


\section{Case Reports in Gastroenterology}

(c) 2018 The Author(s). Published by S. Karger AG, Basel
www.karger.com/crg

Heubi and Bishop: Long-Term Cholic Acid Treatment in a Patient with Zellweger Spectrum Disorder

\section{Author Contributions}

J.E.H. provided care for the patient, provided patient history data and liver chemistry data and interpretation, helped draft the manuscript, and critically revised the manuscript for content. J.E.H. serves as guarantor for this article, accepts full responsibility for the work, had access to the data, and controlled the decision to publish. W.B.P. provided care for the patient, provided patient history data, helped draft the manuscript, and critically revised the manuscript for content.

\section{References}

1 Waterham HR, Ferdinandusse S, Wanders RJ. Human disorders of peroxisome metabolism and biogenesis. Biochim Biophys Acta. 2016 May;1863(5):922-33.

2 Berendse K, Klouwer FC, Koot BG, Kemper EM, Ferdinandusse S, Koelfat KV, et al. Cholic acid therapy in Zellweger spectrum disorders. J Inherit Metab Dis. 2016 Nov;39(6):859-68.

3 Klouwer FC, Berendse K, Ferdinandusse S, Wanders RJ, Engelen M, Poll-The BT.Zellweger spectrum disorders: clinical overview and management approach. Orphanet J Rare Dis. 2015 Dec;10(1):151.

4 Wanders RJ, Ferdinandusse S. Peroxisomes, peroxisomal diseases, and the hepatotoxicity induced by peroxisomal metabolites. Curr Drug Metab. 2012 Dec;13(10):1401-11.

5 Ferdinandusse S, Denis S, Faust PL, Wanders RJ. Bile acids: the role of peroxisomes. J Lipid Res. 2009 Nov;50(11):2139-47.

6 Setchell KD, Bragetti P, Zimmer-Nechemias L, Daugherty C, Pelli MA, Vaccaro R, et al. Oral bile acid treatment and the patient with Zellweger syndrome. Hepatology. 1992 Feb;15(2):198-207.

7 Heubi JE, Bove KE, Setchell KD. Oral cholic acid is efficacious and well tolerated in patients with bile acid synthesis and Zellweger spectrum disorders. J Pediatr Gastroenterol Nutr. 2017 Sep;65(3):321-6.

8 Warren M, Mierau G, Wartchow EP, Shimada H, Yano S. Histologic and ultrastructural features in early and advanced phases of Zellweger spectrum disorder (infantile Refsum disease). Ultrastruct Pathol. 2018 MayJun;42(3):220-7.

9 Heimbach JK, Kulik LM, Finn RS, Sirlin CB, Abecassis MM, Roberts LR, et al. AASLD guidelines for the treatment of hepatocellular carcinoma. Hepatology. 2018 Jan;67(1):358-80.

10 Keane MH, Overmars H, Wikander TM, Ferdinandusse S, Duran M, Wanders RJ, et al. Bile acid treatment alters hepatic disease and bile acid transport in peroxisome-deficient PEX2 Zellweger mice. Hepatology. 2007 Apr;45(4):982-97.

11 Baes M, Van Veldhoven PP. Mouse models for peroxisome biogenesis defects and $\beta$-oxidation enzyme deficiencies. Biochim Biophys Acta. 2012 Sep;1822(9):1489-500.

12 Dirkx R, Vanhorebeek I, Martens K, Schad A, Grabenbauer M, Fahimi D, et al. Absence of peroxisomes in mouse hepatocytes causes mitochondrial and ER abnormalities. Hepatology. 2005 Apr;41(4):868-78.

13 Krysko 0, Hulshagen L, Janssen A, Schütz G, Klein R, De Bruycker M, et al. Neocortical and cerebellar developmental abnormalities in conditions of selective elimination of peroxisomes from brain or from liver. J Neurosci Res. 2007 Jan;85(1):58-72.

14 Faust PL, Banka D, Siriratsivawong R, Ng VG, Wikander TM. Peroxisome biogenesis disorders: the role of peroxisomes and metabolic dysfunction in developing brain. J Inherit Metab Dis. 2005;28(3):369-83.

15 Heubi JE, Setchell KD, Bove KE. Long-term cholic acid therapy in Zellweger spectrum disorders. Case Rep Gastroenterol. 2018 Jun;12(2):360-72. 


\section{Case Reports in Gastroenterology}

\section{Case Rep Gastroenterol 2018;12:661-670}

DOI: $10.1159 / 000494555$

(c) 2018 The Author(s). Published by S. Karger AG, Basel www.karger.com/crg

Heubi and Bishop: Long-Term Cholic Acid Treatment in a Patient with Zellweger Spectrum Disorder
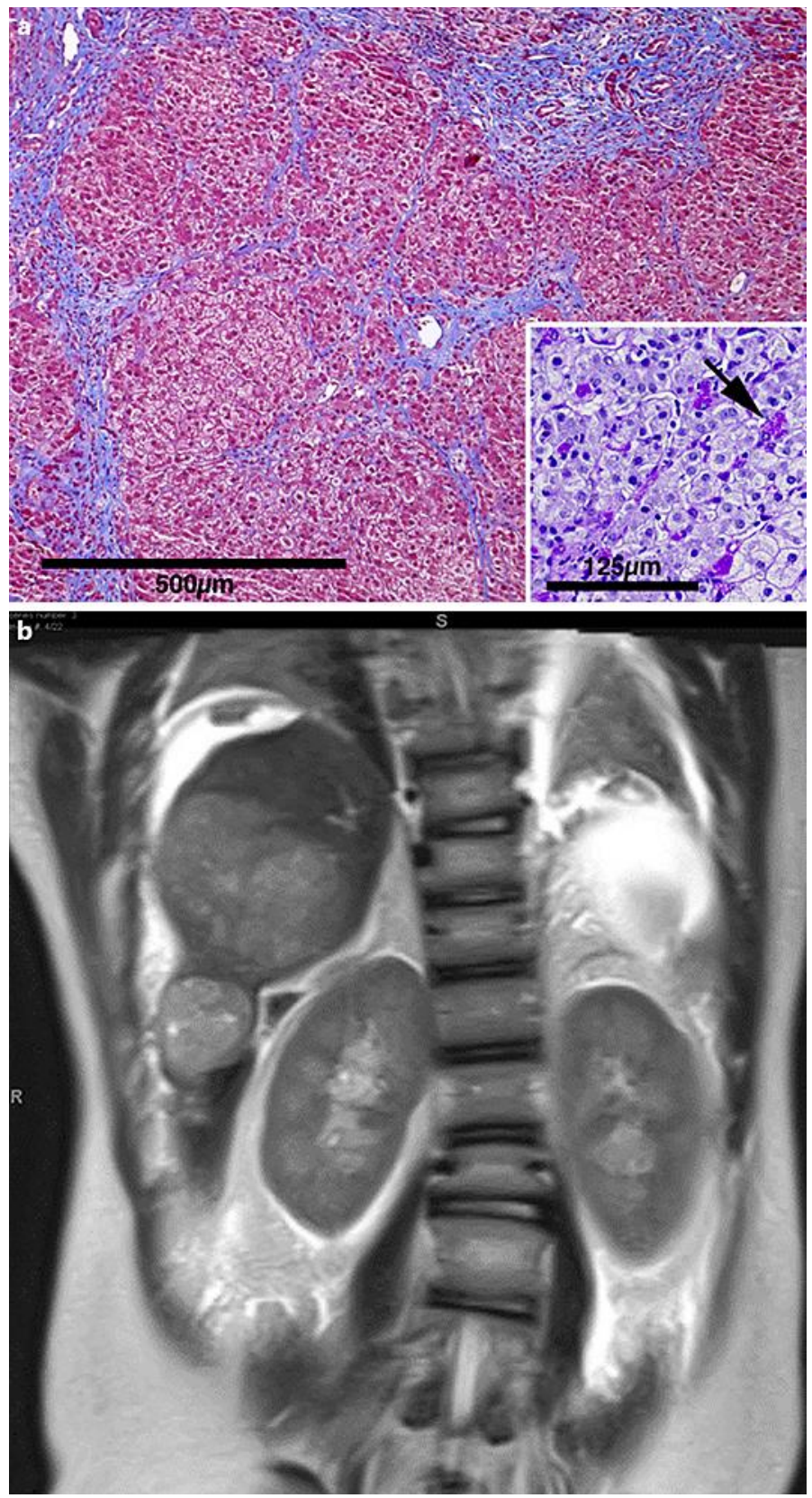

Fig. 1. a Liver biopsy at age 2 years shows periportal fibrosis with septa formation and pericellular fibrosis. Hepatocytes are slightly swollen and inflammation is minimal. Masson Trichrome stain. Inset shows scattered enlarged sinusoidal macrophages containing PAS+ inclusions. Diastase-PAS stain. b MRI image reveals small liver containing two well-defined tumor masses interpreted as HCC. 


\section{Case Reports in Gastroenterology}

(c) 2018 The Author(s). Published by S. Karger AG, Basel www.karger.com/crg

Heubi and Bishop: Long-Term Cholic Acid Treatment in a Patient with Zellwege Spectrum Disorder

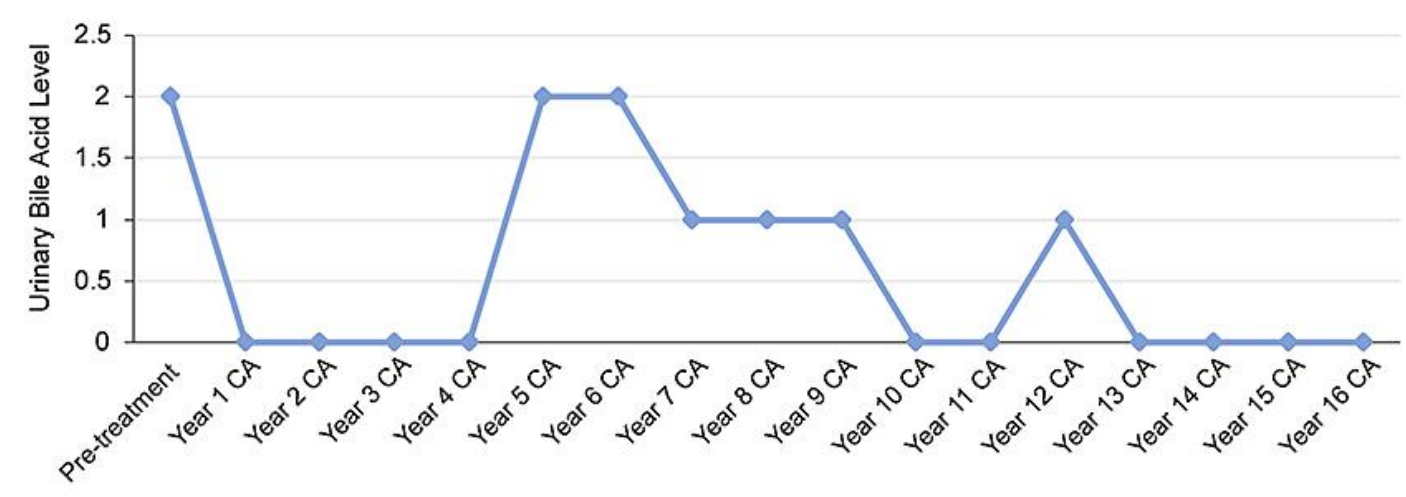

Highest Urinary Bile Acid Level During Year: $0=$ Normal; 1 = Slight/Mild Elevation; 2 = Significant/Moderate Elevation

Fig. 2. Highest level of urinary bile acid during each year of cholic acid (CA) treatment. Urinary bile acid was assessed $\geq 1$ time each year through 16 years of treatment. The highest value obtained in each year is shown. The FAB-MS mass spectra at baseline and post-treatment were assessed as normal (score 0) or as showing slight (score 1), significant (score 2), or marked (score 3) increases in the levels of atypical bile acids [7]. 
Heubi and Bishop: Long-Term Cholic Acid Treatment in a Patient with Zellweger

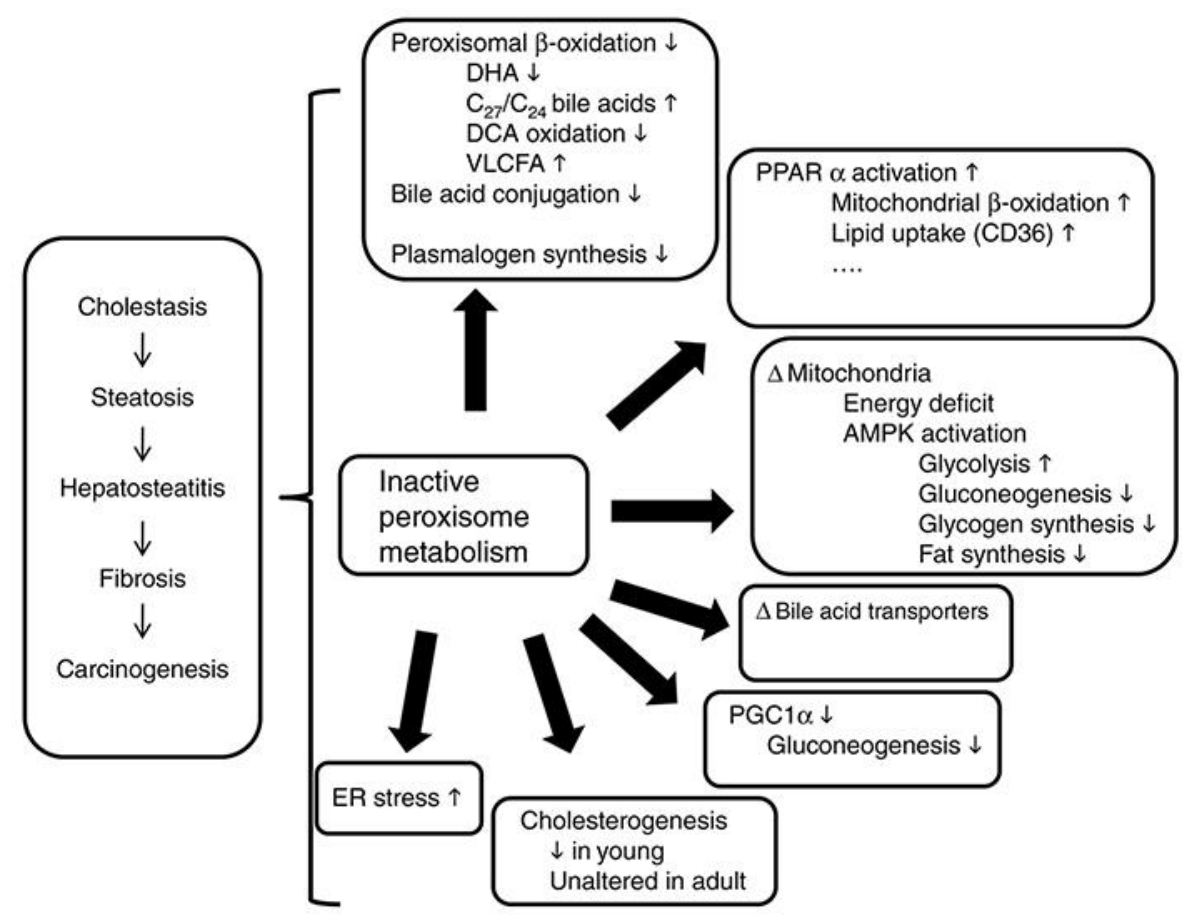

Fig. 3. Overview of metabolic abnormalities reported in liver of mice with peroxisome biogenesis defects. Reprinted with permission from Baes and Van Veldhoven [11].AMPK, AMP-activated protein kinase; DCA, dichloroacetate; DHA, docosahexaenoic acid; ER, endoplasmic reticulum; PGC1 $\alpha$, peroxisome proliferatoractivated receptor gamma coactivator 1-alpha; PPAR $\alpha$, proliferator-activated receptor alpha; VLCFA, very long chain fatty acids. 


\section{Case Reports in Gastroenterology}

\begin{tabular}{l|l}
\hline Case Rep Gastroenterol 2018;12:661-670 \\
\hline DOI: 10.1159/000494555 & $\begin{array}{l}\text { @ 2018 The Author(s). Published by S. Karger AG, Basel } \\
\text { www.karger.com/crg }\end{array}$ \\
\hline
\end{tabular}

Heubi and Bishop: Long-Term Cholic Acid Treatment in a Patient with Zellweger Spectrum Disorder

Table 1. Serum liver chemistries during long-term cholic acid therapy in a patient with Zellweger spectrum disorder

\begin{tabular}{|c|c|c|c|c|c|c|c|}
\hline & $\begin{array}{l}\text { ALT, } \\
\mathrm{U} / \mathrm{L} \text { (RR) }\end{array}$ & $\begin{array}{l}\text { AST, } \\
\mathrm{U} / \mathrm{L} \text { (RR) }\end{array}$ & $\begin{array}{l}\text { ALP, } \\
\mathrm{U} / \mathrm{L}(\mathrm{RR})\end{array}$ & $\begin{array}{l}\text { GGT, } \\
\mathrm{U} / \mathrm{L}(\mathrm{RR})\end{array}$ & $\begin{array}{l}\text { Total bilirubin, } \\
\mathrm{mg} / \mathrm{dL} \text { (RR) }\end{array}$ & $\begin{array}{l}\text { PT, } \\
\text { s (RR) }\end{array}$ & INR \\
\hline Pre-CA (age 3-5 months) & $184(4-35)$ & $299(10-50)$ & $560(82-383)$ & $191(12-122)$ & $0.8(0.2-1.0)$ & $11(9-13)$ & 1.0 \\
\hline Pre-CA (age 1 year, 4 months) & $257(4-35)$ & $146(10-50)$ & $349(82-383)$ & $73(1-39)$ & $0.5(0.2-1.0)$ & $12.4(9-13)$ & 1.2 \\
\hline 5-month CA therapy & $95(5-30)$ & $220(10-50)$ & $379(104-345)$ & $49(3-22)$ & $0.4(0.2-1.0)$ & NA & NA \\
\hline 2-year 9-month CA therapy & $49(5-20)$ & NA & NA & $124(3-22)$ & $0.6(0.2-1.0)$ & $12(11-14)$ & 1.0 \\
\hline 7-year 3-month CA therapy & $44(0-39)$ & $69(0-33)$ & $223(<300)$ & NA & $0.7(0.2-1.0)$ & $13.5(10.1-13.1)$ & 1.1 \\
\hline 12-year 9-month CA therapy & $79(30-65)$ & $97(15-37)$ & $274(52-171)$ & $243(15-85)$ & $0.7(0.0-1.0)$ & $12(9.0-11.4)$ & 1.2 \\
\hline 14-year 9-month CA therapy & $99(30-65)$ & $91(15-37)$ & $474(50-136)$ & $233(15-85)$ & $0.9(0.0-1.0)$ & NA & NA \\
\hline 16-year 11-month CA therapy & $71(<78)$ & $76(<37)$ & $245(<117)$ & NA & $0.9(0.0-1.0)$ & $13.1(9.4-12.9)$ & 1.2 \\
\hline 18-year 6-month CA therapy & $92(0-41)$ & NA & $309(40-129)$ & $278(8-61)$ & $3.8(<1.2)$ & $12(9-12)$ & 1.2 \\
\hline
\end{tabular}

ALP, alkaline phosphatase; ALT, alanine aminotransferase; AST, aspartate aminotransferase; CA, cholic acid; GGT, gamma -glutamyl transpeptidase; INR, international normalized ratio; NA, not available; PT, prothrombin time; RR, reference range. Bolded val ues are above the reference range. 